\title{
LETTER
}

\section{A Simple Criterion for Nonrotating Reference Frames} Peter Collas $]^{1}$ and David Klein ${ }^{2}$

\begin{abstract}
We prove a theorem that gives an easily verifiable necessary and sufficient condition for a reference frame with fixed spacelike coordinates to be nonrotating in the sense of Walker. Applications are discussed.
\end{abstract}

KEY WORDS: Nonrotating frames; Fermi-Walker transport.

\footnotetext{
${ }^{1}$ Department of Physics and Astronomy, California State University, Northridge, Northridge, CA 91330-8268. Email: peter.collas@csun.edu.

${ }^{2}$ Department of Mathematics, California State University, Northridge, Northridge, CA 91330-8313. Email: david.klein@csun.edu.
} 


\section{INTRODUCTION}

Walker [1] defined the reference frame of a nonrotating observer as a frame in which the acceleration of a free test particle in the neighborhood of the observer is independent of its velocity. This definition is consistent with the corresponding definition in Newtonian mechanics, and is useful in general relativity. Nonrotating frames are essential tools in descriptions of relativistic precession [2] and frame dragging [3].

Walker showed that an orthonormal tetrad, at each point on a timelike path $\mathbf{x}(t)$, formed by the unit tangent vector to $\mathbf{x}$ and the unit vectors in the directions of the space axes, is nonrotating when each of the vectors in the tetrad satisfies Eq. (1) below.

In this paper we prove a theorem that gives a simple necessary and sufficient condition for a coordinate frame to be nonrotating on a spacetime path $\mathbf{x}(t)$ whose space coordinates are fixed and whose time coordinate varies. The coordinate frame consists of the tangent vectors of the coordinates in which the metric is given. Our theorem gives simple criteria for this coordinate frame to be nonrotating at a given fixed point in space. The assumption that the space coordinates are fixed along the spacetime path is not as restrictive as it might appear. By transforming to a new coordinate system, which is moving relative to the old one, a point with fixed spatial coordinates in the new system corresponds to a path in space in the old system. Fermi coordinates along a geodesic provide such an example which we discuss in relation to our theorem in Section 3 below. We also illustrate how the theorem may be used by identifying a nonrotating frame for the (interior) van Stockum [4] metric for an infinite rotating dust cylinder. In Section 2 we state and prove the theorem.

\section{THEOREM ON NONROTATING FRAMES}

The Fermi-Walker equations for a vector $\xi^{\alpha}$ are

$$
\nabla_{\vec{u}} \xi^{\alpha}=-\Omega_{\beta}^{\alpha} \xi^{\beta},
$$

where $\vec{u}$ is the four-velocity, $\Omega^{\alpha}{ }_{\beta}=a^{\alpha} u_{\beta}-u^{\alpha} a_{\beta}$, and $a^{\alpha}$ is the four-acceleration. Throughout, Greek indices take values from the set $\{0,1,2,3\}$, while Latin indices take values from the set $\{1,2,3\}$.

Eq. (1) may be rewritten as

$$
\frac{d \xi^{\alpha}}{d \tau}=-\left(\Gamma_{\beta \gamma}^{\alpha} u^{\gamma}+\Omega_{\beta}^{\alpha}\right) \xi^{\beta},
$$

where $\tau$ is proper time. Let $x^{0}=t, x^{1}, x^{2}, x^{3}$ be coordinates for a chart $U$ of spacetime. We consider the trajectory in spacetime given by $\mathbf{x}(t)=$ $\left(t, x_{0}^{1}, x_{0}^{2}, x_{0}^{3}\right)$, where $x_{0}^{1}, x_{0}^{2}, x_{0}^{3}$ are the coordinates of a fixed point in space and $\left(t, x_{0}^{1}, x_{0}^{2}, x_{0}^{3}\right) \in U$ for all $t$ in an open interval $I$ on the real line

Assume the following conditions on the metric $g=g_{\alpha \beta} d x^{\alpha} d x^{\beta}$ : 
(a) For the metric $g$ on $\mathbf{x}(t)$ we have that

$g(\mathbf{x}(t))=g_{00}(\mathbf{x}(t))\left(d x^{0}\right)^{2}+g_{11}(\mathbf{x}(t))\left(d x^{1}\right)^{2}+g_{22}(\mathbf{x}(t))\left(d x^{2}\right)^{2}+g_{33}(\mathbf{x}(t))\left(d x^{3}\right)^{2}$,

where $g_{00}(\mathbf{x}(t))<0$ and the other three coefficients are positive, for $t \in I$. In other words, we assume that $g_{\alpha \beta}(\mathbf{x}(t))=0$ when $\alpha \neq \beta$ (though $g_{\alpha \beta}$ may be nonzero on $U-\{\mathbf{x}(t)\})$ and that the coordinate $x_{0}=t$ is the time coordinate on $\mathbf{x}(t)$.

(b) $g_{\alpha \beta, 0}=0$ on the path $\mathbf{x}(t)$ for all $\alpha$ and $\beta$. In other words, the metric is stationary.

In more concise terms these conditions require $g$ to be diagonal and stationary on the spacetime path $\mathbf{x}(t)$. With these assumptions, the four-velocity of the observer $\mathbf{x}(t)$ may now be expressed as $\vec{u}=\left(d t / d \tau, d x_{0}^{1} / d \tau, d x_{0}^{2} / d \tau, d x_{0}^{3} / d \tau\right)=$ $\left(1 / \sqrt{-g_{00}(\mathbf{x}(t))}, 0,0,0\right)$.

Theorem: Assume that the metric $g$ satisfies conditions (a) and (b) on $\mathbf{x}(t)$. Then the orthonormal coordinate frame given by $\vec{u}$ together with the three spacelike vectors $1 / \sqrt{g_{i i}(\mathbf{x}(t))} \partial / \partial x^{i}$ is non rotating along the path $\mathbf{x}(t)$ (in the sense that each vector field satisfies the Fermi-Walker equations) if and only if $g_{i 0, j}=g_{j 0, i}$ on $\mathbf{x}(t)$ for all $i, j=1,2,3$.

Remark The Theorem may be reformulated to say that the coordinate tangent vectors form a nonrotating orthonormal frame if and only if the curl of the vector $\left(g_{10}, g_{20}, g_{30}\right)$ vanishes, i.e., if and only if the one-form $g_{i 0} d x^{i}$ is closed on $\mathbf{x}(t)$.

The proof of the theorem depends on the following observations and a lemma. If $g$ satisfies (a) and (b), then on $\mathbf{x}(t)$

$$
\begin{aligned}
\Gamma_{\beta \gamma}^{\alpha} & =\frac{1}{2} g^{\alpha \mu}\left(g_{\mu \beta, \gamma}+g_{\mu \gamma, \beta}-g_{\beta \gamma, \mu}\right) \\
& =\frac{1}{2} g^{\alpha \alpha}\left(g_{\alpha \beta, \gamma}+g_{\alpha \gamma, \beta}-g_{\beta \gamma, \alpha}\right), \quad \text { (no sum) } .
\end{aligned}
$$

The relations below follow immediately:

$$
\begin{aligned}
\Gamma_{j 0}^{0} & =\frac{1}{2} g^{00} g_{00, j}, \\
\Gamma^{j}{ }_{00} & =-\frac{1}{2} g^{j j} g_{00, j}, \\
\Gamma^{i}{ }_{i 0} & =0 .
\end{aligned}
$$

Lemma: If $g$ satisfies conditions (a) and (b) on $\mathbf{x}(t)$, then:

(1) For all $\alpha$,

$$
\frac{d u^{\alpha}}{d \tau}=0
$$


(2) The acceleration components are given by

$$
a^{\alpha}=-\frac{\Gamma_{00}^{\alpha}}{g_{00}} .
$$

(3) On $\mathbf{x}(t)$ the tensor $\Omega^{\alpha}{ }_{\beta}=a^{\alpha} u_{\beta}-u^{\alpha} a_{\beta}$, satisfies:

$$
\begin{aligned}
& {\Omega^{0}}_{0}=0, \\
& {\Omega^{\alpha}}^{\alpha}=0, \text { unless } \alpha \text { or } \beta=0, \\
& \Omega^{0}{ }_{j}=-g_{j j} u^{0} a^{j} .
\end{aligned}
$$

Proof of the Lemma. For part (1) observe that,

$$
\frac{d u^{\alpha}}{d \tau}=\frac{\partial u^{\alpha}}{\partial x^{\mu}} \frac{\partial x^{\mu}}{\partial \tau}=\frac{\partial u^{\alpha}}{\partial x^{0}} u^{0}=0,
$$

because $u^{0}=1 / \sqrt{-g_{00}}$ does not depend on $x^{0}=t$ and the other components of $\vec{u}$ are zero. Part (2) follows from

$$
a^{\alpha}=\frac{d u^{\alpha}}{d \tau}+\Gamma_{\beta \gamma}^{\alpha} u^{\beta} u^{\gamma}=\Gamma_{00}^{\alpha} u^{0} u^{0},
$$

where the second equation is a consequence of part (1). For part (3), observe that because $g$ is diagonal on $\mathbf{x}(t), \Omega^{0}{ }_{0}=a^{0} u_{0}-u^{0} a_{0}=g_{00}\left(a^{0} u^{0}-u^{0} a^{0}\right)=0$. The tensor $\Omega^{\alpha}{ }_{\beta}=a^{\alpha} u_{\beta}-u^{\alpha} a_{\beta}$ is zero unless $\alpha$ or $\beta=0$ because $u^{j}$ and $u_{j}$ vanish. Finally, $\Omega^{0}{ }_{j}=a^{0} u_{j}-u^{0} a_{j}=-u^{0} a_{j}=-g_{j j} u^{0} a^{j}$.

Proof of the Theorem. It is easy to check that any four-velocity automatically satisfies the Fermi-Walker equations. In component form, the spacelike vectors of the tetrad are $\left(0,1 / \sqrt{g_{11}}, 0,0\right),\left(0,0,1 / \sqrt{g_{22}}, 0\right),\left(0,0,0,1 / \sqrt{g_{33}}\right)$. Choose any one of these vectors and denote it by $\vec{\xi}=\left(\xi^{0}, \xi^{1}, \xi^{2}, \xi^{3}\right)$. We first show that if $g$ satisfies conditions (a) and (b), then

$$
\frac{d \xi^{0}}{d \tau}=-\left(\Gamma_{\beta \gamma}^{0} u^{\gamma}+\Omega^{0}{ }_{\beta}\right) \xi^{\beta} .
$$

Each step of the calculation below follows from Eqs. (4) - (6), the Lemma, or 
from fact that $g^{j j}=1 / g_{j j}$ for a diagonal matrix $g$.

$$
\begin{aligned}
-\left(\Gamma_{\beta \gamma}^{0} u^{\gamma}+\Omega^{0}{ }_{\beta}\right) \xi^{\beta} & =-\Gamma_{\beta 0}^{0} u^{0} \xi^{\beta}-\Omega^{0}{ }_{j} \xi^{j} \\
& =-\Gamma_{j 0}^{0} u^{0} \xi^{j}+g_{j j} u^{0} a^{j} \xi^{j} \\
& =\left(-\Gamma^{0}{ }_{j 0}+g_{j j} a^{j}\right) u^{0} \xi^{j} \\
& =\left(-\frac{1}{2} g^{00} g_{00, j}-g_{j j} \frac{\Gamma^{j}{ }_{00}}{g_{00}}\right) u^{0} \xi^{j} \\
& =\left(-\frac{1}{2} g^{00} g_{00, j}+g_{j j} \frac{\frac{1}{2} g^{j j} g_{00, j}}{g_{00}}\right) u^{0} \xi^{j} \\
& =0=\frac{d \xi^{0}}{d \tau} .
\end{aligned}
$$

For the spatial components $(j=1,2,3)$,

$$
d \xi^{j} / d \tau=0
$$

Eq. (16) follows from the same argument as in the proof of part 1 of the Lemma. On the other hand, using Eqs. (4) - (6), the Lemma, and $\xi^{0}=0$, we find that,

$$
\begin{aligned}
-\left(\Gamma_{\beta \gamma}^{j} u^{\gamma}+\Omega_{\beta}^{j}\right) \xi^{\beta} & =-\Gamma_{\beta 0}^{j} u^{0} \xi^{\beta}-\Omega^{j}{ }_{\beta} \xi^{\beta} \\
& =-\Gamma_{\beta 0}^{j} u^{0} \xi^{\beta}-\Omega^{j}{ }_{0} \xi^{0} \\
& =-\Gamma^{j}{ }_{i 0} u^{0} \xi^{i} \\
& =\frac{1}{2} g^{j j}\left(g_{j i, 0}+g_{j 0, i}-g_{i 0, j}\right) u^{0} \xi^{i} \\
& =\frac{1}{2} g^{j j}\left(g_{j 0, i}-g_{i 0, j}\right) u^{0} \xi^{i} .
\end{aligned}
$$

In view of Eqs. (16) and (17), the Fermi-Walker equations (2) are satisfied on $\mathbf{x}(t)$ for all $\alpha$ if and only if $g_{j 0, i}-g_{i 0, j}=0$ for all $i, j$. This concludes the proof of the theorem.

\section{THE VAN STOCKUM METRIC}

We illustrate the above theorem by applying it to the van Stockum metric [2]. The van Stockum solution represents a rotating dust cylinder of infinite extent along the axis of symmetry (z-axis) but of finite radius. We consider only the interior solution which in coordinates rotating with the dust particles takes its simplest form below:

$$
d s^{2}=-F d t^{2}+L d \phi^{2}+2 M d t d \phi+H d r^{2}+H d z^{2},
$$


where,

$$
F=1, \quad L=r^{2}\left(1-a^{2} r^{2}\right), \quad M=a r^{2}, \quad H=e^{-a^{2} r^{2}} .
$$

In Eqs. (19), $0 \leq r \leq R$ for a constant $R$ that determines the radius of the cylinder, and $a$ is the angular velocity of the dust particles.

We now perform a rotation to noncomoving coordinates by the transformation

$$
t=\bar{t}, \quad \phi=\bar{\phi}-\Omega \bar{t}, \quad r=\bar{r}, \quad z=\bar{z} .
$$

In the barred coordinates, the metric coefficients are:

$$
\bar{F}=F+2 \Omega M-\Omega^{2} L, \quad \bar{L}=L, \quad \bar{M}=M-\Omega L, \quad \bar{H}=H .
$$

In order to eliminate the coordinate singularity at $\bar{r}=0$, we change to Cartesian coordinates by the transformation

$$
\bar{r}^{2}=x^{2}+y^{2}, \quad \tan \bar{\phi}=\frac{y}{x}, \quad d \bar{r}=\frac{x d x+y d y}{\sqrt{x^{2}+y^{2}}}, \quad d \bar{\phi}=\frac{x d y-y d x}{x^{2}+y^{2}} .
$$

Substituting and collecting terms we obtain,

$$
\begin{aligned}
d s^{2} & =-\bar{F} d t^{2}+\left(\frac{\bar{L}}{r^{4}} y^{2}+\frac{\bar{H}}{r^{2}} x^{2}\right) d x^{2}+\left(\frac{\bar{L}}{r^{4}} x^{2}+\frac{\bar{H}}{r^{2}} y^{2}\right) d y^{2} \\
& +2\left(\frac{\bar{H}}{r^{2}}-\frac{\bar{L}}{r^{4}}\right) x y d x d y \\
& +2 \frac{\bar{M}}{r^{2}} x d t d y-2 \frac{\bar{M}}{r^{2}} y d t d x+\bar{H} d z^{2}
\end{aligned}
$$

and using Eqs. (21) we write

$$
\begin{aligned}
d s^{2} & =-\left(F+2 \Omega M-\Omega^{2} L\right) d t^{2}+\left(\frac{L}{r^{4}} y^{2}+\frac{H}{r^{2}} x^{2}\right) d x^{2} \\
& +\left(\frac{L}{r^{4}} x^{2}+\frac{H}{r^{2}} y^{2}\right) d y^{2}+2\left(\frac{H}{r^{2}}-\frac{L}{r^{4}}\right) x y d x d y \\
& +2 \frac{(M-\Omega L)}{r^{2}} x d t d y-2 \frac{(M-\Omega L)}{r^{2}} y d t d x+H d z^{2} .
\end{aligned}
$$

Eqs. (19) may be substituted into this last expression, and the limits as $r \rightarrow 0$ of each of the coefficients exists and they give the metric along the axis of symmetry. It is easily established that this metric is diagonal on the axis of symmetry and in fact it equals the Minkowski metric on that axis, so conditions (a) and (b) are satisfied for all $\Omega$. An easy calculation shows that on the $z$-axis,

$$
g_{t x, y}=-g_{t y, x}=\Omega-a .
$$


By our theorem, the frame on the axis given by $\partial / \partial t, \partial / \partial x, \partial / \partial y, \partial / \partial z$ is nonrotating if and only if $\Omega=a$. This result was noted in [4] from direct calculation using the Fermi-Walker equation, and a limiting argument.

We note that the hypotheses of our theorem are satisfied by Fermi coordinates for an observer moving along a geodesic, as one would expect since the reference frame is inertial and nonrotating by construction. On a geodesic, the metric is Minkowskian in Fermi coordinates, and the connection coefficients are zero along the path. It follows immediately that all first order partial derivatives of the metric coefficients vanish [5], and hence our condition $g_{i 0, j}=g_{j 0, i}$ is satisfied trivially.

\section{ACKNOWLEDGMENT}

The authors wish to thank Professor John Lawrence for helpful comments.

\section{REFERENCES}

[1] Walker, A. G. (1935). Proc. Edin. Math. Soc. 4, 170.

[2] Hamilton, J. D. (1996). Am. J. Phys. 64, 1197.

[3] Collas, P. and Klein, D. (2004). Gen. Rel. Grav. 36, 1197.

[4] van Stockum, W. J. (1937). Proc. R. Soc. Edin. 57, 135.

[5] Misner, C. W., Thorne, K. S., and Wheeler, J. A. (1973). Gravitation, W. H. Freeman, San Francisco, p. 331. 